\title{
Biodiversidad de macroalgas en arrecifes coralinos de la Sonda de Campeche, el Caribe Mexicano y Belice
}

\section{Macroalgae biodiversity in coral reefs of the Campeche Sound, the Mexican Caribbean and Belize}

\author{
Alicia GonzÁlez-Solis ${ }^{1}$, Daniel Torruco*1 \& Ángel Daniel Torruco-GonzÁlez ${ }^{2}$ \\ ${ }^{1}$ Centro de Investigación y de Estudios Avanzados del Instituto Politécnico Nacional. Unidad Mérida, A.P. 73 Cordemex, 97310 \\ Mérida, Yucatán, México. \\ ²Universidad Anahuac-Mayab. Carretera Mérida-Progreso, km 15.5 C.P. 97310, Mérida Yucatán, México. \\ *dantor6660@gmail.com
}

\begin{abstract}
RESUMEN
Se analizan las coberturas de macroalgas de 24 arrecifes coralinos de la Sonda de Campeche, el Caribe Mexicano y Belice. La mayoría de las 73 especies corresponden a la flora tropical y si bien un gran número de ellas posee una distribución reducida existen representantes cosmopolitas y pan-tropicales. Dada la escasez de datos cuantitativos de macroalgas en estas zonas, en el presente trabajo se analiza la importancia de algunos atributos ecológicos en su distribución regional. Los muestreos involucran registros de 1 a $50 \mathrm{~m}$ de profundidad mediante fotografía submarina y colecta selectiva. La mayor dominancia la registraron las algas verdes y rojas, las algas pardas y las verde-azules se registraron en menor proporción. Las localidades más diversas según el índice de Simpson fueron: Chemuyil (14,9 bits/ind.) y Punta Allen (7,9 bits/ind.) en el Caribe y Bajo Obispo (7,4 bits/ind.) y Arrecife Alacranes (7,2 bits/ind.) en la Sonda de Campeche. Se registraron altos niveles de afinidad entre las localidades pertenecientes a una misma región, lo cual se discute en función de cada sistema. La distribución espacial dada por el análisis de Componentes Principales muestra también grupos que caracterizan a algún sistema arrecifal.
\end{abstract}

Palabras clave: Algas, distribución, arrecifes coralinos, Sonda de Campeche, Caribe mexicano, Belice.

\begin{abstract}
We analyse macroalgae coverage of 24 coral reefs of the Campeche Sound, Mexican Caribbean and Belize. Most of the 73 species correspond to the tropical flora and even though a large number of them has a reduced distribution there are representatives pan-tropical and cosmopolitan. Given the scarcity of macroalgae quantitative data in these areas, this paper discusses the importance of some ecological attributes in their regional distribution. Those surveys involve records of 1 to $50 \mathrm{~m}$ of depth through underwater photography and selective collects. Greater dominance the green and red algae, recorded it the brown and blue-green algae were recorded to a lesser extent. The sites most different according to the Simpson index were: Chemuyil (14.9 bits/ind.) and Punta Allen (7.9 bits/ind.) in the Caribbean and Bajo Obispo (7.4 bits/ind.) and Arrecife Alacranes (7.2 bits/ind.) in Campeche Sound. High levels of affinity between the sites belonging to the same region, were recorded which is discussed on the basis of each system. The spatial distribution given by the PCA shows also groups that characterized to some system reef.
\end{abstract}

KeYwords: Algae, distribution, coral reefs, Campeche Sound, Mexican Caribbean, Belize.

\section{INTRODUCCIÓN}

La algas marinas de los arrecifes coralinos son comunidades naturales altamente diversas que han desarrollado una organización compleja que se caracteriza por la amplia utilización de nichos ecológicos (Bak 1977, Bula-Meyer 1992); además, desempeñan un papel importante en la dinámica del sistema arrecifal en procesos tanto constructivos como destructivos. No obstante, algunas fanerógamas marinas como Thalassia testudinum K.D. Koening, 1805, Syringodium filiforme Kützing, 1860 y Halodule wrightii Ascherson 1868, ocupan grandes extensiones del fondo en las aguas someras de los arrecifes.

En los arrecifes circundantes a la Península de Yucatán, la vegetación ficológica es muy variada, la mayoría de las especies corresponden a la flora tropical (Callejas et al. 
2005). Así, un gran número de ellas posee una distribución reducida (generalmente caribeña), aunque existen representantes tanto cosmopolitas como pantropicales. Generalmente crecen sobre los $30 \mathrm{~m}$, ya que necesitan la luz para sus procesos vitales; sin embargo, en áreas de aguas extremadamente claras algunas de ellas se pueden encontrar en zonas más profundas (Cetz-Navarro et al. 2008).

Dada la escasez de investigaciones regionales (Ortega et al. 2001, Mateo-Cid et al. 2013), el presente trabajo con la flora ficológica en estas zonas se realizó con el propósito específico de conocer la distribución de las especies y la importancia de ciertos atributos ecológicos en esta zona. Asimismo, se hace una comparación preliminar para dilucidar la posible existencia de patrones de distribución en los diferentes sitios a lo largo de la Península de Yucatán y áreas aledañas, sobre todo considerando que se tienen dos áreas: la Sonda de Campeche y el norte del Caribe, incluyendo localidades de Belice cercanas al borde mexicano.

\section{MATERIALES Y MÉTODOS}

Los datos base del presente trabajo fueron colectados en 24 arrecifes coralinos circundantes a la Península de Yucatán (Fig. 1), durante los años 1995 y 2015; todos los muestreos se realizaron en primavera-verano. La flora corresponde al piso litoral e infralitoral superior. Se utilizó el buceo autónomo como método de colecta cubriendo un intervalo de 1 a $50 \mathrm{~m}$, los datos fueron obtenidos mediante 2 líneas de fototransectos de $20 \mathrm{~m}$ de longitud por cada $10 \mathrm{~m}$ de profundidad paralelos a la línea de costa, acompañados de una pequeña recolección selectiva de las especies encontradas en cada línea; en cada transecto se utilizaban 2 rollos de 30 fotografías. Las fotografías fueron tomadas a cada metro, a $80 \mathrm{~cm}$ del piso utilizando un bastón graduado y una cámara submarina Nikonus V (Liddell \& Ohlhorst 1987). Aun cuando el total de fotografías fue de 7.250, las analizadas fueron 4.173 , las restantes no fueron adecuadas

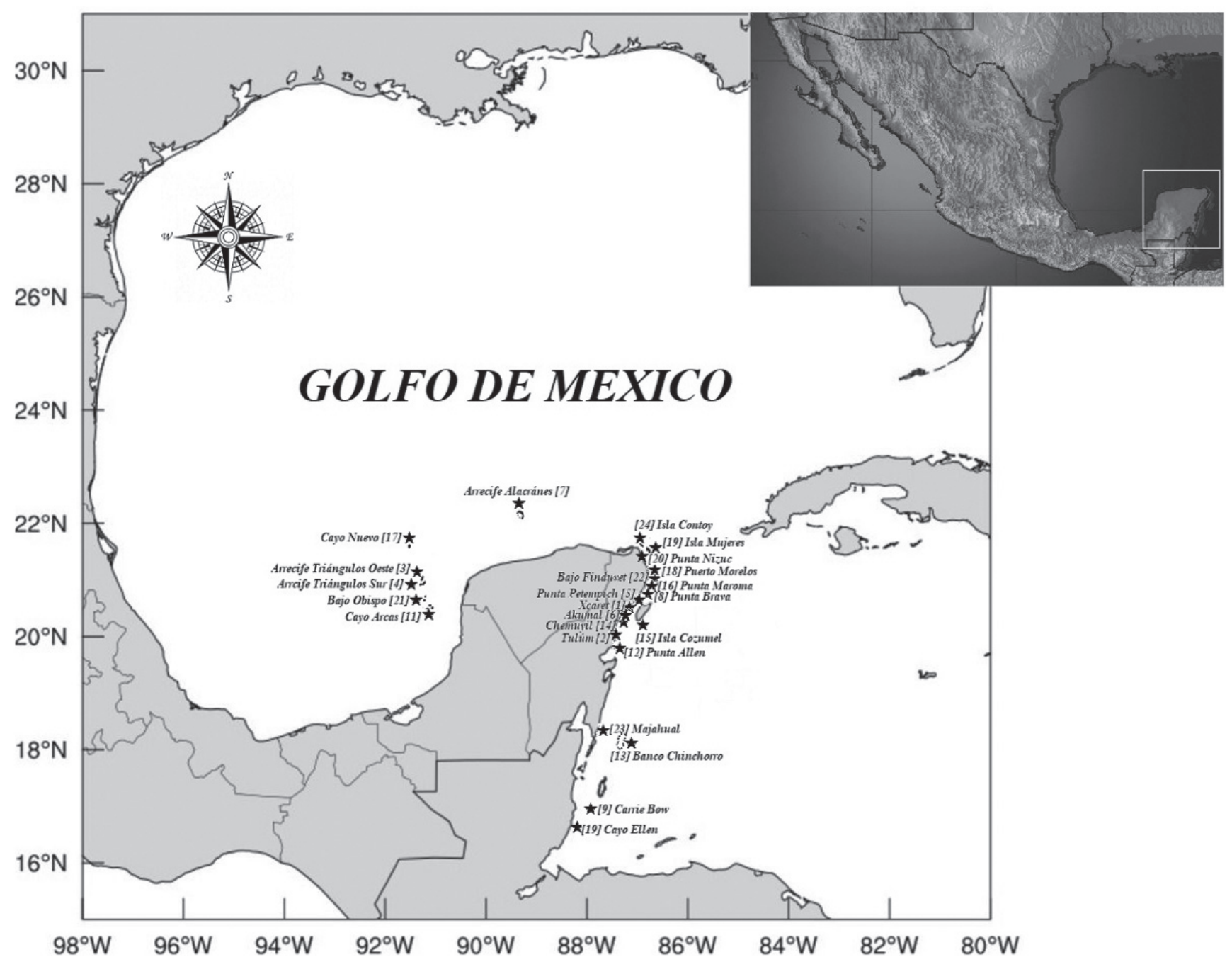

FIGURA 1. Ubicación de los arrecifes coralinos investigados. / Location of coral reefs investigated. 
porque estaban movidas o fuera de foco, en cada una de ellas se obtuvieron las coberturas que correspondían a las especies de macroalgas por la superposición de una rejilla en la fotografía. Los transectos se realizaron donde se encontraba la profundidad requerida sin importar si estaba directamente sobre el arrecife o si caía en alguna zona arenosa, seibadal o restos de coral. Todos los registros están depositados en el Laboratorio de Arrecifes de Coral del CINVESTAV.

Para el análisis de esta comunidad se utilizó como estrategia de primera aproximación un cálculo de macrodescriptores comunitarios: la dominancia de cada especie y la diversidad en cada localidad. El análisis de la dominancia se realizó con base a la propuesta de dominancia Jerárquica de Orlocí (Orlocí 1978). Mientras que la diversidad ecológica fue obtenida mediante el método de Simpson (Pielou 1984), que sigue el siguiente procedimiento:

$$
\text { Diversidad }=\frac{N(N-1)}{\sum_{i} n_{i}\left(n_{i}-1\right)}
$$

Donde: $\mathrm{N}=$ Total de cobertura

$\mathrm{n}_{i}=$ Total de cobertura de la especie $i$

La segunda estrategia fue el uso de estadística multivariada para determinar las afinidades o diferencias entre las localidades. El estudio está basado en un análisis de conglomerados bajo el criterio aglomerativo del Contenido de Información de segundo orden (C.I. ${ }_{2}$ ), codificado para datos cuantitativos de abundancia (Torruco 1995). Posteriormente, se realizó un acercamiento con base en un análisis de Componentes Principales bajo el criterio de varianza-covarianza de la matriz original, para tratar de dilucidar la causalidad y la magnitud de la importancia en la ordenación de esta comunidad (Orlocí 1978).

\section{RESULTADOS}

Se obtuvieron registros de 73 especies de macroalgas y 1 fanerógama marina (Tabla 1). El grupo de las rodofíceas fue el de mayor número de especies y consecuentemente las cianofíceas fueron las menos representadas. En una visión de la dominancia de la comunidad ficológica, Caulerpa sertularoides M.A. Howe 1905, fue la especie con mayor dominancia al presentar $47,03 \%$ del total de todas las especies (Fig. 2). La mayor riqueza de especies se registró en Chemuyil (46 especies) y en Punta Allen (42 especies); mientras que los menores valores de riqueza (1 especie) se registraron en las localidades de Xcaret, Bajo Obispo e Isla Contoy. En cuanto a la diversidad ecológica, Chemuyil en la costa de Quintana Roo en el Caribe, fue la localidad más diversa $(14,97$ bits/ ind.). La mayor equidad corresponde a Punta Nizuc $(0,91)$, también en la costa de ese estado (Tabla 2).

En cuanto al análisis de clasificación entre las localidades, el dendrograma (Fig. 3) muestra a un nivel del $50 \%$ del contenido de información utilizado como similitud entre ellas, tres agrupaciones. El primero es muy heterogéneo e incluye arrecifes de la Sonda de Campeche y el Caribe; sin embargo, presentan un patrón de identidad muy marcado, se unen fuertemente zonas como Tulúm y Xcaret que son arrecifes costeros de la parte media de Quintana Roo y se separan de una asociación formada por arrecifes exclusivos de la Sonda. Este mismo grupo muestra una asociación muy fuerte con dos arrecifes de Belice (Cayo Ellen y Carrie Bow), a la que posteriormente se le adicionan arrecifes costeros e insulares del Caribe. Una subagrupación separa determinantemente Alacranes y Punta Nizuc, así como a Triángulos Oeste y Cayo Nuevo en la Sonda de Campeche. Los otros dos grupos poseen menos elementos con asociaciones no tan fuertes como las subagrupaciones del primero, pero con identidades muy marcadas, ya que forman uniones fuertes que representan, por un lado, arrecifes costeros de la zona norte del Estado de Quintana

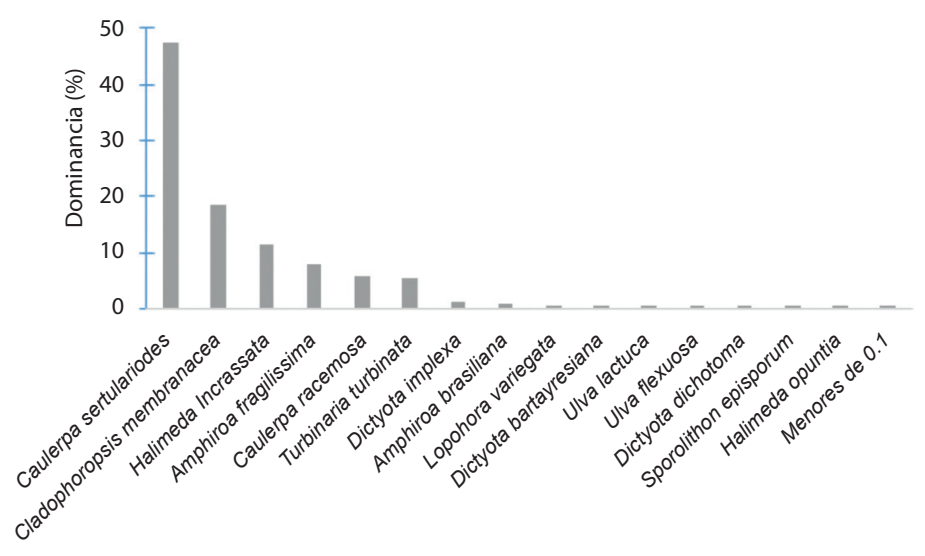

Figura 2. Dominancia en porcentaje de cada una de las especies de macroalgas encontradas en la zona de muestreo. / Dominance as a percentage of each of the species of macroalgae found in the sampling area. 
Roo, como es el caso de Bajo Finduvet, Puerto Morelos y Punta Maroma y por el otro una mezcla de arrecifes costeros e insulares (Punta Petempich, Akumal, Isla Cozumel y Banco Chinchorro), pero todos ellos pertenecientes también a la costa de Quintana Roo en el Caribe Mexicano. Es también notable la relación tan estrecha que guardan los arrecifes de Belice (Cayo Ellen y Carrie Bow) con Majahual y Punta Allen, que son localidades que se ubican en la parte sur de la Península (Fig. 3).
El análisis de componentes principales conforma un grupo compacto con cinco localidades separadas. Estas localidades pertenecen al Caribe y definen características particulares: Banco Chinchorro y Akumal, localizados en el cuadrante superior derecho e inferior derecho respectivamente, son los más alejados. Isla Cozumel y Punta Nizuc tienen una distancia intermedia y Chemuyil es el más cercano al megagrupo. En éste análisis los dos primeros ejes explican el $71 \%$ de la variancia total de los datos (Fig. 4).

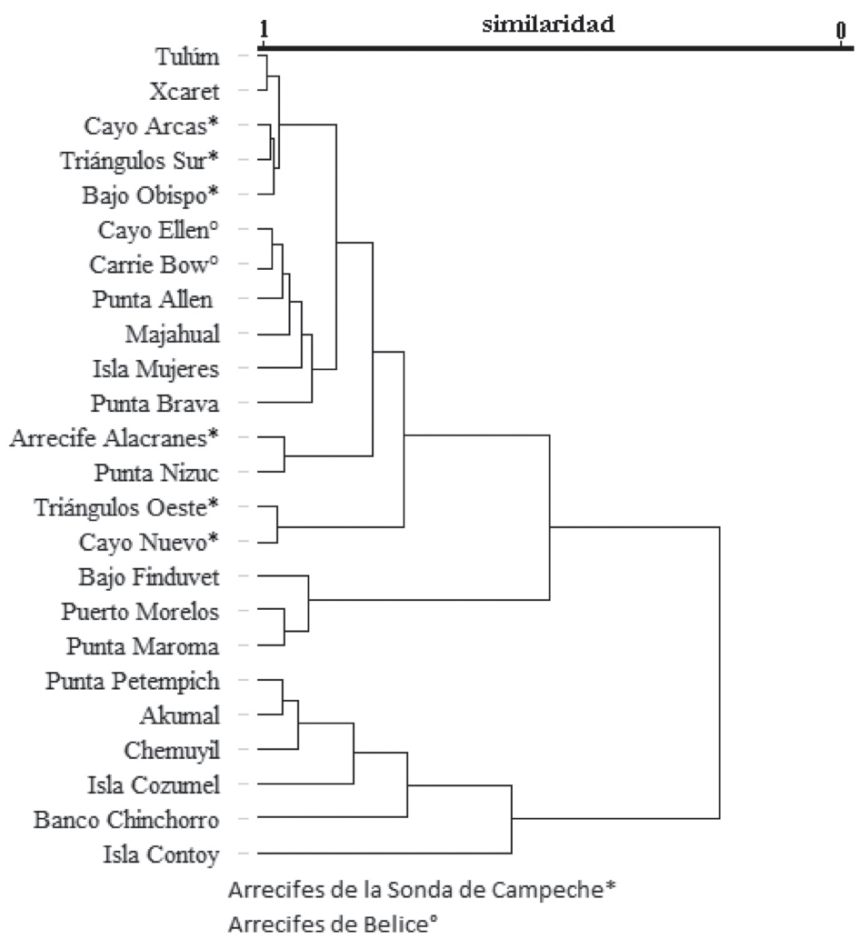

FIgURA 3. Dendrograma que muestra la similitud de las localidades muestreadas. El análisis fue realizado mediante el método aglomerativo del contenido de información. / A dendrogram showing the similarity of the sampled locations. The analysis was carried out using the method Agglomerative of the content of information.

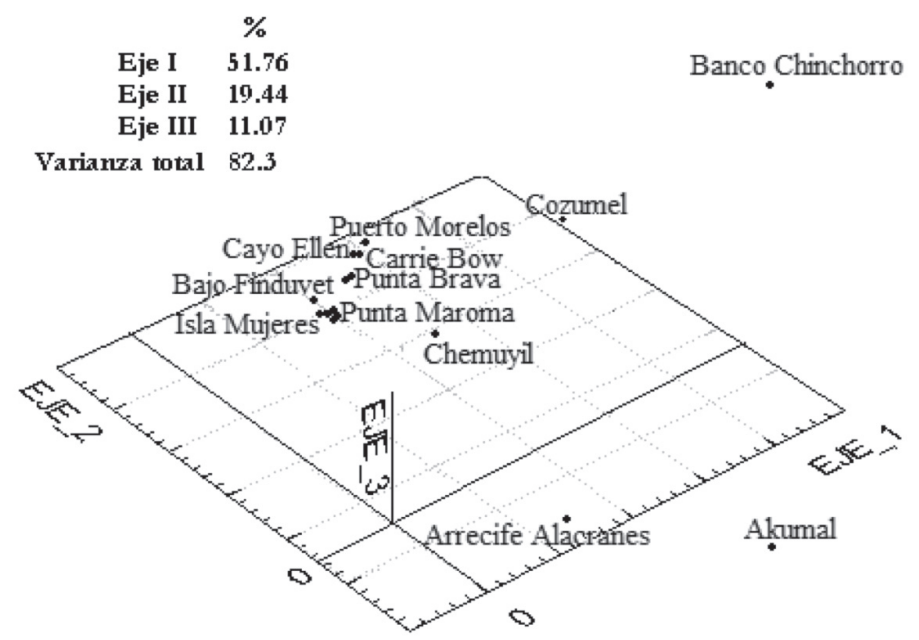

FIgURA 4. Representación tridimensional del ordenamiento de las localidades dado por el Análisis de Componentes Principales. El total de la varianza explicada por los 3 ejes es de $84,3 \%$. / Three-dimensional representation of the sites order given by the PCA. The total of the variance explained by the 3 axes is $84.3 \%$. 
Biodiversidad de macroalgas en arrecifes coralinos de México: GonZÁLEZ-Solis, A. ET AL.

Tabla 1. Presencia de las especies de macroalgas en arrecifes de las Sonda de Campeche, el Caribe Mexicano y Belice. / Macroalgae species presence in the Campeche Sound, the Mexican Caribbean and Belize reefs.

\begin{tabular}{|c|c|c|c|c|c|c|c|c|c|c|c|c|c|c|c|c|c|c|c|c|c|c|c|}
\hline ESPECIES & 1 & 2 & 3 & 4 & 5 & 6 & 7 & 8 & 9 & 10 & 11 & 12 & 13 & 14 & 15 & 16 & 17 & 18 & 19 & 20 & 21 & 22 & 232 \\
\hline Acanthophora spicifera (M.Vahl) Børgesen, 1910 & & & & & & & $\uparrow$ & & & & & & & & & & & & & & & & \\
\hline Amphiroa brasiliana Decaisne, 1842 & & & & & & $\uparrow$ & $\uparrow$ & $\uparrow$ & $\uparrow$ & & & $\uparrow$ & & 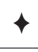 & & & $\uparrow$ & & & & & & \\
\hline Amphiroa fragilissima (Linnaeus) J.V.Lamouroux, 1816 & & & & & & & & & & & & $\hookrightarrow$ & & $\uparrow$ & & & & & & & & & \\
\hline Amphiroa rigida J.V.Lamouroux, 1816 & & $\uparrow$ & & & 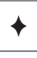 & & $\uparrow$ & & & & & $\uparrow$ & $\uparrow$ & + & $\uparrow$ & & $\uparrow$ & & & & & $\uparrow$ & \\
\hline $\begin{array}{l}\text { Amphiroa tribulus (J.Ellis \& Solander) J.V.Lamouroux, } \\
1816\end{array}$ & & & & & & & & & & & & $\hookrightarrow$ & & $\uparrow$ & & & & $\hookrightarrow$ & & & & & \\
\hline Anadyomene stellata (Wulfen) C.Agardh, 1823 & & & & & $\uparrow$ & & 4 & & & & & & 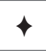 & 4 & & & & & & & & & 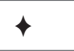 \\
\hline Avrainvillea asarifolia Børgesen, 1909 & & & & & & & & & & & & $\hookrightarrow$ & & & & & & & & & & & \\
\hline Avrainvillea nigricans Decaisne, 1842 & & & & & $\uparrow$ & & & & & & & & & $\uparrow$ & & & & & & & & $\hookrightarrow$ & \\
\hline Avrainvillea rawsonii (Dickie) M.A.Howe, 1907 & & & & & & & & & & & & $\uparrow$ & & 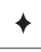 & & & & & & & & & \\
\hline Bostrychia montagnei Harvey, 1853 & & & & & & & & & & & & $\uparrow$ & & & & & & & & & & & \\
\hline $\begin{array}{l}\text { Canistrocarpus cervicornis (Kützing) De Paula \& De } \\
\text { Clerck, } 2006\end{array}$ & & & & & & & $\hookrightarrow$ & & & & & & $\uparrow$ & $\dashv$ & & & & & & & & & \\
\hline Caulerpa cupressoides (Vahl) C.Agardh, 1817 & & & & & & & & & & & & & & & & & $\hookrightarrow$ & & & & & & \\
\hline Caulerpa prolifera (Forsskål) J.V.Lamouroux, 1809 & & & & & & & & & & & & $\hookrightarrow$ & & & & & & & & & & & 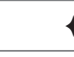 \\
\hline Caulerpa racemosa (Forsskål) J.Agardh, 1873 & & & & & & & $\hookrightarrow$ & & & & & $\hookrightarrow$ & $\hookrightarrow$ & $\hookrightarrow$ & & & $\hookrightarrow$ & $\hookrightarrow$ & & & & & \\
\hline Caulerpa sertularioides (S.G.Gmelin) M.A.Howe, 1905 & & & & & $\uparrow$ & $\uparrow$ & & & & & & $\uparrow$ & & 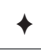 & & & & 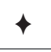 & & & & & \\
\hline Centroceras clavulatum (C.Agardh) Montagne, 1846 & & & & & $\uparrow$ & $\hookrightarrow$ & & & & & & & $\hookrightarrow$ & $\hookrightarrow$ & & & $\hookrightarrow$ & & & & & & \\
\hline Chaetomorpha crassa (C.Agardh) Kützing, 1845 & & & & & & & & & & & & & & & & & & & $\uparrow$ & & & & \\
\hline Chondria capillaris (Hudson) M.J.Wynne, 1991 & & & & & & & & & & & & 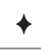 & & & & & & & & & & & \\
\hline Chondria littoralis Harvey, 1853 & & & & & & & & & & & & $\hookrightarrow$ & & & & & & & & & & & \\
\hline $\begin{array}{l}\text { Cladophoropsis membranacea (Hofman Bang ex } \\
\text { C.Agardh) Børgesen, } 1905\end{array}$ & & $\uparrow$ & $\uparrow$ & & & & $\uparrow$ & & & 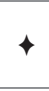 & & $\uparrow$ & & $\hookrightarrow$ & & $\hookrightarrow$ & $\hookrightarrow$ & & $\downarrow$ & & & & \\
\hline Codium decorticatum (Woodward) M.A.Howe, 1911 & & & & & & & & & & & & & & $\uparrow$ & & & & & & & & $\hookrightarrow$ & \\
\hline $\begin{array}{l}\text { Colpomenia sinuosa (Mertens ex Roth) Derbès \& } \\
\text { Solier, } 1851\end{array}$ & & & & & & & & & & & & $\hookrightarrow$ & & & & & & & & & & & \\
\hline Dictyosphaeria cavernosa (Forsskål) Børgesen, 1932 & & $\hookrightarrow$ & 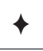 & & & & & & & & & & & $\hookrightarrow$ & 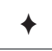 & & $\hookrightarrow$ & & & & & & \\
\hline Dictyota bartayresiana J.V.Lamouroux, 1809 & & $\uparrow$ & & & & & $\hookrightarrow$ & 4 & & & & 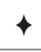 & & $\hookrightarrow$ & & & & & 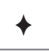 & & & & \\
\hline Dictyota dichotoma (Hudson) J.V.Lamouroux, 1809 & & & & 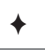 & $\uparrow$ & & & & & & & $\hookrightarrow$ & $\hookrightarrow$ & 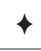 & $\hookrightarrow$ & & & $\hookrightarrow$ & $\hookrightarrow$ & & & 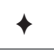 & $\hookrightarrow$ \\
\hline Dictyota implexa (Desfontaines) J.V.Lamouroux, 1809 & & $\hookrightarrow$ & 4 & & 4 & 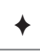 & & $\uparrow$ & & $\hookrightarrow$ & $\uparrow$ & 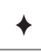 & 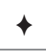 & $\hookrightarrow$ & 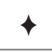 & $\uparrow$ & 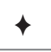 & & $\downarrow$ & $\hookrightarrow$ & & $\hookrightarrow$ & 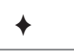 \\
\hline Ectocarpus erectus Kützing, 1845 & & & & & & & & & & & & & & $\hookrightarrow$ & 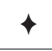 & & & & & & & & \\
\hline Galaxaura J.V.Lamouroux, 1812 & & & & & & & & & & & & & $\hookrightarrow$ & & & & & & & & & & \\
\hline $\begin{array}{l}\text { Ganonema farinosum (J.V.Lamouroux) K.C.Fan \& } \\
\text { Yung C.Wang, } 1974\end{array}$ & & & & & & & & & & & & $\hookrightarrow$ & & & & & & & & & & & \\
\hline Halimeda copiosa Goreau \& E.A.Graham, 1967 & & & & & 4 & & $\hookrightarrow$ & & & & & & $\hookrightarrow$ & $\uparrow$ & & & & & & & & $\hookrightarrow$ & \\
\hline Halimeda discoidea Decaisne, 1842 & & & & 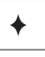 & $\hookrightarrow$ & & 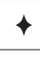 & & & & & $\hookrightarrow$ & $\uparrow$ & 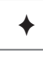 & 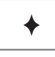 & & & & & & & & $\uparrow$ \\
\hline Halimeda goreaui W.R.Taylor, 1962 & & & & & $\uparrow$ & & $\hookrightarrow$ & & & & & & $\hookrightarrow$ & $\uparrow$ & 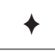 & & $\hookrightarrow$ & & & & & $\hookrightarrow$ & \\
\hline Halimeda incrassata (J.Ellis) J.V.Lamouroux, 1816 & $\uparrow$ & & $\hookrightarrow$ & 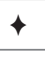 & $\hookrightarrow$ & $\hookrightarrow$ & 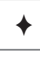 & 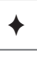 & 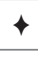 & & $\hookrightarrow$ & 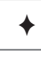 & $\uparrow$ & 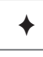 & $\hookrightarrow$ & & 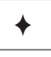 & 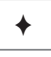 & & & $\hookrightarrow$ & 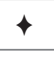 & $\uparrow$ \\
\hline $\begin{array}{l}\text { Halimeda monile (J.Ellis \& Solander) J.V.Lamouroux, } \\
1816\end{array}$ & & & & $\checkmark$ & & & & & & & & & & & & & & & & & & & \\
\hline Halimeda opuntia (Linnaeus) J.V.Lamouroux, 1816 & & & & & 4 & & 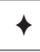 & 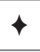 & 4 & & & $\uparrow$ & 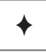 & $\downarrow$ & 4 & 4 & + & + & 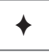 & & & 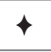 & 4 \\
\hline $\begin{array}{l}\text { Halimeda tuna (J.Ellis \& Solander) J.V.Lamouroux, } \\
1816\end{array}$ & & & & $\hookrightarrow$ & & & & $\hookrightarrow$ & $\uparrow$ & & $\downarrow$ & $\hookrightarrow$ & & $\hookrightarrow$ & $\uparrow$ & & $\uparrow$ & & $\uparrow$ & & & & $\uparrow$ \\
\hline
\end{tabular}


Gayana Bot. 75(1), 2018

\begin{tabular}{|c|c|c|c|c|c|c|c|c|c|c|c|c|c|c|c|c|c|c|c|c|c|c|c|c|}
\hline ESPECIES & 1 & 2 & 3 & 4 & 5 & 6 & 7 & 8 & 9 & 10 & 11 & 12 & 13 & 14 & 15 & 16 & 17 & 18 & 19 & 20 & 21 & 22 & 23 & 24 \\
\hline Hydrolithon boergesenii (Foslie) Foslie, 1909 & & $\hookrightarrow$ & & 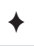 & & & & & & & & & $\hookrightarrow$ & $\hookrightarrow$ & & $\hookrightarrow$ & & & & & & & & \\
\hline Jania adhaerens J.V.Lamouroux, 1816 & & & & & & & & & & & & & $\hookrightarrow$ & & & & & & & & & $\uparrow$ & & \\
\hline Laurencia obtusa (Hudson) J.V.Lamouroux, 1813 & & & & & & & & & & & & $\hookrightarrow$ & & & & & & & & & & & & \\
\hline Lithophyllum prototypum (Foslie) Foslie, 1905 & & $\hookrightarrow$ & & $\hookrightarrow$ & & & $\hookrightarrow$ & & & & & $\hookrightarrow$ & 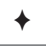 & $\hookrightarrow$ & & & & & & $\hookrightarrow$ & & & & \\
\hline $\begin{array}{l}\text { Lobophora variegata (J.V.Lamouroux) Womersley ex } \\
\text { E.C.Oliveira, } 1977\end{array}$ & & $\hookrightarrow$ & & & $\downarrow$ & $\uparrow$ & 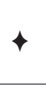 & & & & $\hookrightarrow$ & $\hookrightarrow$ & $\hookrightarrow$ & $\downarrow$ & $\uparrow$ & $\uparrow$ & 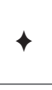 & $\uparrow$ & & & & 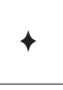 & & \\
\hline Mesophyllum mesomorphum (Foslie) W.H.Adey, 1970 & & + & & & & & & & & & & $\uparrow$ & & 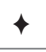 & & $\uparrow$ & & & & & & & & \\
\hline $\begin{array}{l}\text { Neogoniolithon strictum (Foslie) Setchell \& } \\
\text { L.R.Mason, } 1943\end{array}$ & & & & & & & & & & & & $\hookrightarrow$ & & & & & & & & & & & & \\
\hline Neomeris annulata Dickie, 1874 & & & & & & & & & & & & $\hookrightarrow$ & & & & & & & & & & & & \\
\hline Oscillatoria corallinae Gomont ex Gomont, 1890 & & & & & & & & & & & & & & 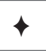 & & & & & & & & & 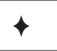 & \\
\hline Padina sanctae-crucis Børgesen, 1914 & & 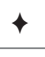 & & & & & & & & & & $\hookrightarrow$ & $\hookrightarrow$ & & & & 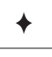 & & & & & & & \\
\hline $\begin{array}{l}\text { Palisada perforata (Bory de Saint-Vincent) K.W.Nam, } \\
2007\end{array}$ & & & & & & & & & & & & $\uparrow$ & & & & & & & & & & & & \\
\hline Penicillus capitatus Lamarck, 1813 & & & & & & & 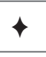 & & & & & $\uparrow$ & & $\hookrightarrow$ & & & & + & & & & 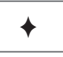 & & \\
\hline Penicillus dumetosus (J.V.Lamouroux) Blainville, 1830 & & & & & & & & & & & & & $\hookrightarrow$ & $\hookrightarrow$ & & & & & & & & $\uparrow$ & & \\
\hline Penicillus pyriformis A.Gepp \& E.S.Gepp, 1905 & & & & & $\uparrow$ & & 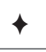 & & & & & $\uparrow$ & $\uparrow$ & $\uparrow$ & & & & & & & & & & \\
\hline Porolithon onkodes (Heydrich) Foslie, 1909 & & & & & & & & & & & & & $\hookrightarrow$ & & & & & & & & & & & \\
\hline Rhipilia tomentosa Kützing, 1858 & & & & & & & & & & & & $\uparrow$ & & & & & & & & & & & & \\
\hline $\begin{array}{l}\text { Rhipocephalus phoenix (J.Ellis \& Solander) Kützing, } \\
1843\end{array}$ & & & & & & $\uparrow$ & & & & & $\hookrightarrow$ & $\hookrightarrow$ & $\hookrightarrow$ & $\downarrow$ & & & $\downarrow$ & & & & & & & \\
\hline Sargassum fluitans (Børgesen) Børgesen, 1914 & & & & & 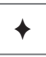 & 4 & $\uparrow$ & & & & & $\hookrightarrow$ & & & & & & & & & & & & \\
\hline Sargassum hystrix J.Agardh, 1847 & & & & & & & $\downarrow$ & & & & & 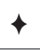 & & & & & & & & & & & & \\
\hline Sargassum vulgare C.Agardh, 1820 & & & & & & & & & & & & 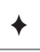 & & $\uparrow$ & & & & & & & & 4 & & \\
\hline 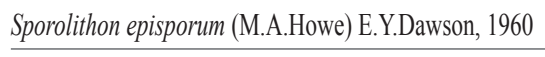 & & 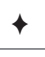 & & & & & & & & & & & & $\uparrow$ & & & & & & & & & & \\
\hline Stypopodium zonale (J.V.Lamouroux) Papenfuss, 1940 & & $\uparrow$ & & & $\uparrow$ & 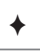 & 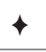 & & & & 4 & 4 & $\uparrow$ & 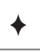 & $\uparrow$ & & & & & & & & & \\
\hline Symploca hydnoides Kützing ex Gomont, 1892 & & & & & & & & & & & & & $\hookrightarrow$ & & & & & & & & & & & \\
\hline Thalassia testudinum K.D.Koenig, 1805 & & & & & & & & & & & & $\hookrightarrow$ & & $\hookrightarrow$ & $\uparrow$ & & $\hookrightarrow$ & & & & & & & \\
\hline $\begin{array}{l}\text { Tricleocarpa fragilis (Linnaeus) Huisman \& } \\
\text { R.A.Townsend, } 1993\end{array}$ & & & & & & & & $\hookrightarrow$ & & & & & 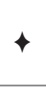 & & & & & & & & & & & \\
\hline Turbinaria turbinata (Linnaeus) Kuntze, 1898 & & & & & $\hookrightarrow$ & & & & & & & 4 & $\hookrightarrow$ & & & & $\hookrightarrow$ & & & & & 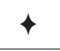 & & \\
\hline Udotea cyathiformis Decaisne, 1842 & & & & & & & & & & & & & $\uparrow$ & 4 & & & & & & & & & & \\
\hline Udotea flabellum (J.Ellis \& Solander) M.A.Howe, 1904 & & & & & $\hookrightarrow$ & & $\hookrightarrow$ & & & & & 4 & & 4 & $\hookrightarrow$ & & 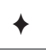 & $\hookrightarrow$ & 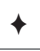 & & & & & \\
\hline Udotea occidentalis A.Gepp \& E.S.Gepp, 1911 & & & & & & & & & & & & 4 & 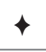 & & & & & & & & & & & \\
\hline Udotea spinulosa M.A.Howe, 1909 & & & & & & & & & & & & & $\hookrightarrow$ & 4 & & & & & & & & 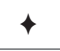 & & \\
\hline Udotea wilsonii A.Gepp, E.S.Gepp \& M.A.Howe, 1911 & & & & & & & & & & & & 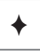 & & & & & & & & & & & & \\
\hline Ulva flexuosa Wulfen, 1803 & & & & & & & & & & & & & 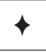 & $\uparrow$ & & & + & & & & & & & \\
\hline Ulva lactuca Linnaeus, 1753 & & & & & & & & & & & & & $\hookrightarrow$ & 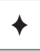 & & & & & & & & & & \\
\hline Valonia macrophysa Kützing, 1843 & & & & & & & & & & & & & + & 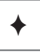 & & & & & & & & & & \\
\hline Valonia ventricosa J.Agardh, 1887 & & & & & $\hookrightarrow$ & $\checkmark$ & 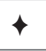 & & & & & 4 & 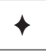 & $\hookrightarrow$ & 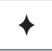 & $\hookrightarrow$ & & $\hookrightarrow$ & & & & & $\hookrightarrow$ & \\
\hline Wrangelia penicillata (C.Agardh) C.Agardh, 1828 & & & & & 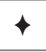 & 4 & & 4 & & & & & & $\uparrow$ & & & & & & & & & & \\
\hline $\begin{array}{l}\text { Yuzurua poiteaui var. gemmifera (Harvey) M.J.Wynne, } \\
2011\end{array}$ & & & & & & & & & & & & & & $\downarrow$ & & & & & & & & & & \\
\hline TOTALES DE ESPECIES & 1 & 12 & 4 & 7 & 20 & 11 & 22 & 8 & 4 & 2 & 6 & 44 & 33 & 46 & 15 & 7 & 19 & 10 & 8 & 2 & 1 & 16 & 9 & 1 \\
\hline
\end{tabular}


TABla 2. Riqueza de especies (S), diversidad (D) y equitatividad (E) de las macroalgas en los arrecifes del sureste de México y Belice según el criterio del índice de Simpson. Se presentan las diversidades máximas y mínimas calculadas a partir de las muestras en los sitios estudiados. / Macroalgae species richness (S), diversity (D) and evenness (E) in the reefs of the southeast of Mexico and Belize according to the criterion of Simpson index. Maximum and minimum diversity calculated from samples in the sites studied are presented.

\begin{tabular}{|c|c|c|c|c|c|c|}
\hline LOCALIDADES & No. & $\mathrm{S}$ & $\mathrm{D}$ & Dmáx & Dmín & $\mathrm{E}$ \\
\hline Xcaret & 1 & 1 & 1.0 & 1.0 & 0.0 & 1.00 \\
\hline Tulúm & 2 & 12 & 6.7 & 12.5 & 1.0 & 0.54 \\
\hline * Triángulos Oeste & 3 & 4 & 1.6 & 4.2 & 1.1 & 0.39 \\
\hline * Triángulos Sur & 4 & 7 & 2.9 & 7.3 & 1.1 & 0.39 \\
\hline P. Petempich & 5 & 20 & 4.4 & 20.1 & 1.0 & 0.22 \\
\hline Akumal & 6 & 11 & 2.9 & 11.0 & 1.0 & 0.27 \\
\hline * A. Alacranes & 7 & 22 & 7.2 & 23.3 & 1.1 & 0.31 \\
\hline P. Brava & 8 & 7 & 2.3 & 7.2 & 1.1 & 0.32 \\
\hline${ }^{\circ}$ Carrie Bow & 9 & 4 & 2.1 & 4.2 & 1.1 & 0.50 \\
\hline${ }^{\circ}$ Cayo Ellen & 10 & 2 & 1.5 & 2.2 & 1.2 & 0.69 \\
\hline * Cayo Arcas & 11 & 6 & 3.1 & 6.8 & 1.3 & 0.46 \\
\hline P. Allen & 12 & 44 & 7.9 & 44.7 & 1.0 & 0.17 \\
\hline B. Chinchorro & 13 & 33 & 6.5 & 33.7 & 1.0 & 0.19 \\
\hline Chemuyil & 14 & 46 & 14.9 & 46.7 & 1.0 & 0.32 \\
\hline I. Cozumel & 15 & 15 & 6.7 & 16.2 & 1.1 & 0.41 \\
\hline P. Maroma & 16 & 7 & 5.0 & 9.4 & 1.8 & 0.53 \\
\hline * Cayo Nuevo & 17 & 19 & 6.9 & 19.5 & 1.0 & 0.35 \\
\hline Pto. Morelos & 18 & 10 & 6.1 & 10.7 & 1.1 & 0.57 \\
\hline I. Mujeres & 19 & 8 & 4.4 & 8.5 & 1.1 & 0.52 \\
\hline P. Nizuc & 20 & 2 & 2.0 & 2.2 & 1.2 & 0.92 \\
\hline * Bajo Obispo & 21 & 16 & 7.4 & 17.3 & 1.1 & 0.43 \\
\hline Bajo Finduvet & 22 & 9 & 3.7 & 9.2 & 1.0 & 0.40 \\
\hline Majahual & 23 & 1 & 1.0 & 1.0 & 1.0 & 1.00 \\
\hline TOTAL & & 73 & 13.5 & 73.4 & 1.0 & 0.18 \\
\hline
\end{tabular}

* Arrecifes de la Sonda de Campeche.

- Arrecifes costeros de Belice.

\section{DISCUSIÓN}

Los resultados muestran que la flora marina de los arrecifes coralinos de la Península de Yucatán es muy diversa. Garduño-Solorzano et al. (2005), analizan bibliográficamente, la distribución geográfica de algas verdes, registrando 169 especies en 49 géneros, donde la mayor riqueza corresponde a Cladocera Latreille 1829 y Caulerpa Lamouroux 1809. De sus datos propios analizan 25 localidades con 69 especies, pero confina la provincia de la ficoflora tropical a un intervalo de Veracruz hasta Quintana Roo, sin diferenciar los dos sistemas: el Golfo de México y el Caribe Mexicano. Es posible que, al considerar solo zonas costeras, la identidad de los dos sistemas se diluya y pueda integrarse como un todo, como lo visualizaron Garduño-Solorzano et al. (2005), para las clorofíceas. Por otro lado, pocas publicaciones abordan el estudio de macroalgas de profundidades entre $0-50 \mathrm{~m}$ del infralitoral (Lehman \& Tunnell 1992, Mendoza-González et al. 2000). En nuestro caso registramos 73 especies de macroalgas, de 
las tres clases; a pesar de coincidir que Caulerpa fue la más dominante, si encontramos evidencias que ambos sistemas tienen identidad propia. En casi todas las localidades existe una combinación de substratos (rocoso y arenoso), sobre todo en las partes someras de las áreas arrecifales, lo que proporciona una gran variedad de hábitats. Sin embargo, la mayoría de las especies se presentan en localidades que poseen una cierta protección al oleaje. Suárez (1973) señala para Cuba 116 taxa, en la zona infralitoral.

Hillebrand (2004) menciona que la diversidad global sigue un gradiente latitudinal, alta en los trópicos y que desciende hacia los polos; no obstante, las macroalgas son la excepción al ser más diversas en regiones templadas (Santelices et al. 2009). Los arrecifes estudiados pertenecen a la región tropical y en esta zona no existen grandes variaciones de temperatura ni de salinidad, ya que por un lado las variaciones de latitud son pequeñas y en lo segundo la naturaleza kárstica de la Península de Yucatán, sin ríos superficiales no propicia grandes fluctuaciones: por consiguiente, el componente tropical es el mejor representado en la diversidad ficológica (Miloslavich et al. 2010). Garduño-Solorzano et al. (2005), encuentran un gradiente latitudinal norte-sur de diversidad, mencionando que la mayoría de la flora presenta una distribución continua pero también que se presentan algunas discontinuidades y que la mayor riqueza la sitúan en Quinta Roo, lo cual coincide con nuestros datos. Sin embargo, encontramos una fuerte identidad entre las localidades con respecto a las macroalgas, ya que éstas se separan determinantemente debido a dos aspectos: sistema oceánico (Golfo de México y Mar Caribe) y su cercanía a la costa (arrecifes costeros y arrecifes oceánicos) e incluso se podría mencionar que en el caso de los arrecifes de Quintana Roo, se diferencian arrecifes al norte y arrecifes al sur del estado.

Los factores que controlan la abundancia de macroalgas en el Caribe han sido discutidos por varios autores (Huerta \& Garza 1980, Littler \& Littler 1984), donde mencionan que las diferencias en las comunidades algales pueden estar relacionadas a diversos factores, tanto ambientales como antropogénicos. Sin embargo, pocos datos cuantitativos están disponibles. El resumen estadístico para las áreas arrecifales puede presentar una subapreciación y una posible distorsión de los esquemas de cambio en los ecosistemas arrecifales (Goreau \& Goreau 1973, Steneek \& Dethier 1994). En nuestro caso, se tienen varios elementos: los arrecifes de la Sonda de Campeche son arrecifes oceánicos, aislados y que presentan interacciones de pesca y de explotación de hidrocarburos en sus áreas (Yáñez-Arancibia \& Day 2004), sus aguas son ya la mezcla de aguas del Caribe que penetra por el canal de Yucatán y las propias del Golfo (Vidal et al. 1989), mientras que en los arrecifes del Caribe el impacto lo representa el turismo, sobre todo en la parte norte (GonzálezSolís \& Torruco 2015).

Algunos de los patrones de distribución de las algas, pueden ser atribuidos a los efectos del ambiente, que pueden afectar el reclutamiento y la supervivencia de las especies. Martínez et al. (2012) y Bartsch et al. (2012) mencionan que los límites de distribución de múltiples especies de macroalgas sugieren que las temperaturas extremas pueden limitar la diversidad y que estas tendencias también pueden estar relacionadas a factores como la disponibilidad de luz, sedimentación alta y especificidad del substrato, ya que estos elementos podrían ser los de mayor importancia en estas comunidades (Bradbury \& Young 1981, Littler et al. 1987). Desafortunadamente el presente análisis no involucra un examen escrupuloso de estos factores, debido a que este trabajo se realiza exclusivamente en arrecifes coralinos, donde la disponibilidad de luz está regulada por el crecimiento arrecifal formando mosaicos de luzobscuridad, pero donde la penetración es alta, el substrato es generalmente duro y la sedimentación es baja con excepción de eventos esporádicos (tormentas y huracanes). Pianka (1966) menciona que las interacciones bióticas son más importantes que las condiciones ambientales que pueden ser más o menos estables y que direccionan gradientes de diversidad a grandes escalas. En los arrecifes, la competencia con corales y la presencia de ramoneadores pueden inhibir la diversidad de las macroalgas en los trópicos (Bolton 1994).

Desde hace varios años, observaciones en otros arrecifes han empezado a sugerir que los arrecifes no son estables en sus asociaciones intraespecíficas en un supuesto equilibrio, como se postulaba anteriormente (Ohlhorst \& Liddell 1988). En el área estudiada, una diferencia obvia entre las principales islas y las áreas costeras de Quintana Roo son los impactos ecológicos asociados con la urbanización. El principal perjuicio de la contaminación incluye la sedimentación causada por las escorrentías y la eutrofización de bahías debidas a las descargas de drenaje (Rappaport et al. 1985, Littler et al. 1993). Por ejemplo, este impacto origina un alto número de cianofíceas en Isla Cozumel.

La flora ficológica en los arrecifes coralinos presenta asociaciones debidas a atributos principales como son: el piso, la facie, las interacciones bióticas y la protección al oleaje. Estos atributos podrían ser los que originan las principales diferencias de diversidad y por consiguiente los responsables de las asociaciones interespecíficas que existen en las comunidades algales del sureste de México, como podría inferirse del análisis de Componentes Principales, en donde la cantidad de varianza que explican los dos primeros ejes de ordenación reflejan la magnitud de las fuerzas que deben controlar la distribución de la abundancia de las macroalgas. Una fuente de incertidumbre son las características del estado de las especies que pueden originar pares asociados pero que no deben presentar ninguna importancia en la distribución a grandes niveles. Esto podría ser causado por la relativa constancia de los factores del ambiente en las regiones tropicales y sobre todo por los atributos de estado que son marcadamente estacionales y no 
se presentan como una característica siempre constante en la flora ficológica.

Así, el substrato y la protección física de la línea costera al oleaje son en este caso las fuerzas controladoras de la distribución regional de la flora ficológica. Estos factores juegan un papel importante en el control de los patrones y magnitudes de la producción primaria en los arrecifes de coral. Por otro lado, luz, sedimentación y régimen de olas están altamente relacionados con la profundidad y son también parámetros probables a ejercer una fuerte influencia en la distribución de las algas. Keith et al. (2014) mencionan que la temperatura superficial del mar (TSM) es el direccionador primario de la distribución de la diversidad, por su coincidencia con isotermas de verano e invierno (Luning 1990) en zonas templadas, pero que en el trópico predominan las interacciones bióticas.

Un aspecto importante que debe tomarse en cuenta en la fuerte identidad de sitios que pertenecen al Caribe son los efectos de eventos meteorológicos de fuerte impacto (tormentas tropicales y huracanes), ya que presentan gran influencia en lo relacionado a la disponibilidad y a la alta inestabilidad del substrato. Dicho disturbio causa grandes cambios en las poblaciones de algas bénticas, debido tanto a efectos directos como al intenso oleaje, como a efectos indirectos, como es el aumento de sedimentos en suspensión que eventualmente serán depositados sobre la flora (Tunnichffe 1983, Boem 1988). Algunas especies como Halimeda se caracterizan por presentar un sistema rizoidal y una morfología compacta, lo que le confiere cierta ventaja para resistir oleajes intensos (Matta 1981), otras sobreviven a la abrasión del sedimento en áreas con gran energía, debido a su capacidad para colonizar hábitats crípticos, como grietas o fisuras o en algunos casos un crecimiento en la base de corales duros. En algunas localidades las corrientes que se registran son tan fuertes como para eliminar por su acción a las macroalgas junto con su substrato como ha ocurrido en otras localidades del Caribe (Matta 1981); bajo el influjo de las fuerzas desatadas por las tormentas tropicales y los ciclones, el suelo marino parece materialmente lijado, dejando al descubierto la roca madre (obsv. pers.). Posteriormente, en estas zonas, la colonización por algas fue mucho más activa, al no tener competidores con esa capacidad de respuesta.

Estos disturbios catastróficos eliminan temporalmente competidores, organismos herbívoros y otros que actúan como un control ecológico sobre las especies oportunistas, en estas áreas se observa una espectacular sucesión algal; esto podría ser la causa de que algunas localidades presenten una afinidad pareada con áreas muy cercanas, como es el caso de los arrecifes costeros. Además, los disturbios catastróficos originan un movimiento de las comunidades bénticas y de sedimentos, causando una súbita suspensión de cantidades considerables de nutrientes en el mar, consecuentemente este proceso resulta en un aprovechamiento intenso por las algas y por consiguiente una rápida proliferación (Crossland et al. 1984, Lapointe 1995). En el área de la costa de Quintana Roo se han observado patrones de recolonización diferentes después del paso de un ciclón (como fue el caso de los huracanes Mitch en 1998 y Dean en 2007) y que dan lugar a diferencias florísticas entre las áreas con daños máximos y las zonas con pocos efectos, como son los casos del sur del Estado de Quintana Roo y los arrecifes de la Sonda de Campeche.

La posición geográfica en la zona correspondiente a la Sonda de Campeche en el Golfo de México es importante por las condiciones fluctuantes de temperatura del agua. Este factor puede afectar directa e indirectamente la distribución de las algas y tener influencia con los patrones de diversidad y su variación local. Un aspecto particular que se debe tener en cuenta es la naturaleza de parche (tanto espacial como temporal) de muchas especies de algas, que puede mostrar un decremento progresivo en la cobertura algal hacia la costa. Esto último puede aparecer como un resultado parcialmente originado por el decremento en las tasas de crecimiento debido principalmente a la baja disponibilidad de nutrientes, sobre todo por la naturaleza kárstica de la Península de Yucatán. Gordillo et al. (2012) mencionan que el crecimiento de las macroalgas está limitado por los nutrientes (nitrógeno, fosfatos y bicarbonatos), y Santelices et al. (2009) mencionan que la diversidad a escala regional parece estar influenciada por este factor. En nuestro caso, y específicamente para la Sonda de Campeche, los arrecifes de coral son áreas pobres en nutrientes (oligotróficos); no obstante, la cercanía a desembocaduras de ríos importantes pueden imponer un cierto ritmo de crecimiento a las macroalgas al poner a disponibilidad nutrientes.

Earle (1972) menciona que la flora algal del Golfo de México tiene afinidades geográficas similares a los corales y consideraciones en la distribución de corales escleractinios (Dustan \& Halas 1987, Torruco 1995), podría dar evidencia del efecto de este grupo en el crecimiento y supervivencia de las macroalgas. En Jamaica, al tener una sobreexplotación pesquera, los invertebrados herbívoros (especialmente erizos) son los responsables de los patrones que se presentan en la estructura del fitobentos (Liddell \& Ohlhorst 1987), un aspecto similar ocurre en los arrecifes costeros de Belice, donde existe una sobreexplotación de peces herbívoros. En las costas mexicanas, las poblaciones de escáridos y acantúridos, se encuentran en un estado casi natural sin un efecto de sobreexplotación como sucede en las costas de Jamaica y Belice, al parecer estos organismos son los responsables, en gran parte, del pastoreo que ocurre en los arrecifes coralinos y que configuran en gran medida la estructura del fitobentos. En conclusión, los límites de distribución regional de las macroalgas en los arrecifes de coral de la Sonda de Campeche, el Caribe Mexicano y la parte aledaña de Belice, están controlados por factores ambientales, competencias (espacio y luz) con otros 
componentes del ecosistema arrecifal y por herbivoría (peces e invertebrados), como lo mencionan Hatcher (1985) y Mumby (2009). La importancia relativa de los determinantes bióticos y los físicos en la estructura de la comunidad algal béntica es evidente. Los diferentes sistemas oceanográficos (el Golfo de México y el Mar Caribe) son identificados por elementos de la flora ficológica, separándolos determinantemente.

\section{AGRADECIMIENTOS}

Se hace un reconocimiento al Consejo Nacional de Ciencia y Tecnología por el apoyo otorgado para la realización de varios proyectos en los que se incluyeron numerosas exploraciones. Así mismo se hace un reconocimiento a la Secretaría de Marina y al Gobierno del Estado de Campeche, por el apoyo en el traslado del personal hacia los diferentes arrecifes de la Sonda de Campeche.

\section{REFERENCIAS}

BAK, R.P.M. 1977. Coral reefs and their zonation in Netherlands Antilles. In: Frost, S.H., Weiss, M.P., Saunders, J.B. (eds.), Reef and related Carbonates ecology and sedimentology, pp. 4: 316. American Association Petroleum Geology. Studies in Geology.

Bartsch, I., Wiencke, C., Laepple, T. 2012. Global seaweed biogeography under a changing climate: the prospected effects of temperature. In: Wiencke, C., Bischof K. (eds.), Seaweed biology, pp. 383-406. Springer-Verlag, Berlin.

Boem, R.M. 1988. Recognition of storm impact on the reef sediment record. Proceeding of the 6th International Coral Reef Symposium, Australia. 2: 475-478.

Bolton, J.J. 1994. Global seaweed diversity: patterns and anomalies. Botanica Marina 37: 241-245.

Bradbury, R.H., Young, P.C. 1981. The effects of a major forcing function, wave energy, on a coral reef ecosystem. Marine Ecology Progress Series 5: 229-241.

Bula-Meyer, G. 1992. ¿Están las algas marinas de los fondos plano-arenosos profundos fisiológicamente restringidas a estos hábitats continuos al talud arrecifal? Memorias VIII semana Nacional de Ciencia y Tecnología del Mar, Santa Marta, Colombia 1: 196-204.

Callejas-Jiménez, M.E., Sentíes-Granados, A., Dreckmann, K.M. 2005. Macroalgas bentónicas de Puerto Real, Faro Santa Rosalía y Playa Preciosa, Campeche, México, con algunas consideraciones florísticas y ecológicas para el estado. Hidrobiológica 15(1): 89-96.

Cetz-Navarro, N.P., Espinoza-Avalos, J., Sentíes-Granados, A., QuAn-Young, L. I. 2008. Nuevos registros de macroalgas para el atlántico mexicano y riqueza florística del Caribe mexicano. Hidrobiológica 18(1): 11-19.

Crossland, C.J., Hatcher, B.G., Atkinson, M.J., Smith, S.V. 1984. Dissolved nutrients of the high latitude coral reef Hourtman Abrolhos Islands, Western Australia. Marine Biological Progress Series. 14:159-163.
Dustan, P., Halas, J.C. 1987. Changes in the reef coral community of Carysfort Reef, Key Largo, Florida: 19741982. Coral Reefs 6: 91106.

EARLE, S. A. 1972. Benthic algae and seagrasses species in the Gulf of Mexico. In: Bushnell, V.C. (ed.), Serial atlas of the marine environments, pp. 25-29. American Geographical Society, New York.

Garduño-Solórzano, G., Godínez-Ortega, J.L., Ortega, M.M. 2005. Distribución geográfica y afinidad por el substrato de las algas (Cholorophyceae) bénticas de las costas mexicanas del Golfo de México y mar Caribe. Boletín de la Sociedad Botánica de México 76: 61-78.

GonzÁlez-Solís, A., Torruco, D. 2015. Perfiles sociales y económicos en la costa de Quintana Roo: su relación con el turismo de playa. Investigación Ambiental 7(1): 1-11.

GordiLlo, F.J.L. 2012. Environment and algal nutrition. In: Wiencke, C., Bischof, K. (eds.), Seaweed biology, pp. 6786. Springer-Verlag, Berlin.

Goreau, T.F., Goreau. N.I. 1973. Ecology of Jamaican coral reefs. II. Geomorphology, zonation and sedimentary phases. Bulletin Marine Science 23: 399-464.

Hatcher, B.G. 1985. Ecological research at the Houtman's Abrolhos: High latitude reefs of Western Australia. Proceeding Fifth International Coral Reef Congress, Tahiti 6: 291-297.

Hillebrand, H. 2004. On the generality of the latitudinal diversity gradient. The American Naturalist 163: 192-211.

Huerta, L., Garza, A. 1980. Contribución al conocimiento de la flora marina de la zona sur del litoral de Quintana Roo, México. Anales Escuela Nacional de Ciencias Biológicas. México 23: 25-44.

Keith, S.A., Kerswell, A.P., Connolly, S.R. 2014. Global diversity of marine microalgae: Environmental conditions explain less variation in the tropics. Global Ecology and Biogeography 23: 517-529.

LAPOINTE, B.E. 1995. A comparison of nutrient limited productivity in Sargassum natans from neritic versus oceanic waters of the Western North Atlantic Ocean. Limnology and Oceanography 40: 625-633.

Lehman, R.L., Tunnell, W. J. JR. 1992. Species composition and ecology of the microalgae of Enmedio Reef, Veracruz, Mexico. Texas Journal of Science 44: 445-457.

Liddell, W.D., Ohlhorst, S.L. 1987. Patterns of reef community structure, North Jamaica. Bulletin Marine Science 40(2): 311-329.

Littler, M.N., Littler, D.S. 1984. Models of tropical reef biogenesis: The contribution of algae. Progress Phycology Research 3: 323-364.

LitTler, M.N., TaYlor, P.R., Littler, D.S., Sims, R.H., NorRis, J.N. 1987. Dominant macrophyta standing stocks, productivity and community structure on a Belizean Barrier Reef. Atoll Research Bulletin 302: 1-24.

Littler, M.N., Littler, D.S., Lapointe, B.E. 1993. Modification of tropical reef community structure due to cultural eutrophication: The southwest coast of Martinique. Proceeding 7th International Coral Reef Symposium. Guam 1: 335-343.

LÜNING, K. 1990. Seaweeds: their environment, biogeography, and ecophysiology. Wiley \& Sons, Toronto, Canada. 527 pp.

Martinez, B., Arenas, F., Rubal, M., Burgues, S., Esteban, 
Biodiversidad de macroalgas en arrecifes coralinos de México: GonZÁLEz-Solis, A. ET AL.

R., Garcia-Plazaola, I., Figueroa, F. L., Pereira, R., Saldana, L., Sousa-Pinto, I., Trilla, A., Viejo, R.M. 2012. Physical factors driving intertidal macroalgae distribution: physiological stress of a dominant fucoid at its southern limit. Oecologia 170: 341-353.

MatTA, J.L. 1981. Disturbio y sucesión ecológica en las poblaciones de macroalgas en un arrecife coralino del mar Caribe. VII Symposio Latinoamericano sobre Oceanografía Biológica. Acapulco, México. 499-520.

Mateo-Cid, L.E., Mendoza-González, A.C., Ávila-Ortiz, A.G., Díaz-Martínez, S. 2013. Algas marinas bentónicas del litoral de Campeche, México. Acta Botánica Mexicana 104: 53-92.

Mendoza-González, A.C., Mateo-Cid, L.E., Searles, R.B. 2000. New records of benthic marine algae from Isla Cozumel, México. Phaeophyta and Chlorophyta. Bulletin of Marine Science 66: 119-130.

Miloslavich, P., Díaz, J.M., Klein, E., Alvarado, J.J., Díaz, C., Gobín, J., Escobar, E., Cruz-Motta, J.J., Weil, E., Cortéz, J., Bastidas, A. C., Robwertson, R., Zapata, F., Martín, A., Castillo, J., Kazandian, A., Ortíz, M. 2010. Marine biodiversity in the Caribbean: Regional estimates and distribution patterns. Plos One 5(8): 1-25.

Mumby, P.J. 2009. Phase shifts and the stability of macroalgal communities on Caribbean coral reefs. Coral Reefs 28: 761-773.

Ohlhorst, S.L., Liddell, W.D. 1988. The effect of substrata microtopography on reef community structure, $60120 \mathrm{~m}$. Proceeding of the 6th International Coral Reef Symposium, Australia 3: 355-360.

OrLocí, L. 1978. Multivariate analysis in vegetation research. 2nd. Edition. Dr. W.J. Junk Publisher, The Hague 452 pp.

Ortega, M.M., Godínez, J.L., Garduño-Solórzano, G. 2001. Catálogo de algas bénticas de las costas del Golfo de
México y mar Caribe. Universidad Nacional Autónoma de México. México, D.F., México. 594 pp.

PianKA, E.R. 1966. Latitudinal gradients in species diversity: a review of concepts. The American Naturalist 100: 33-46.

Pielou, E.C. 1984. The interpretation of ecological data: A premier on classification and ordination. Wiley Interscience Publications, New York. 263 pp.

RAPPAPORT, D.J., Regier, W.A., Hutchinson, T.C. 1985. Ecosystem behaviour under stress. American Naturalist. 125: 617640 .

Santelices, B., Bolton, J.J., Meneses, I. 2009. Marine algal communities. In: Witman, J.D., Roy, K. (eds.), Marine Macroecology, pp. 153-192. University of Chicago Press, Chicago, IL.

Steneek R.S., Dethier, M.N. 1994. A functional group approach to the structure of algal dominated communities. Oikos 69: 16-34.

SuÁrez, A.M. 1973. Catálogo de algas cubanas. Universidad de la Habana, La Habana. 35 pp.

Torruco, D. 1995. Faunística y Ecología de los corales escleractinios en los arrecifes de coral del sureste de México. Thesis PhD. Universidad de Barcelona, España. $423 \mathrm{pp}$.

TunNichfFe, V. 1983. Caribbean staghorn coral population: prehurricane Allen condition in Discovery Bay, Jamaica. Bulletin Marine Science 33: 132-151.

Vidal, V.M.V., Vidal, F.V., Pérez-Molero, J.M. 1989. Atlas Oceanográfico del Golfo de México. Instituto de Investigaciones Eléctricas, Serie Atlas IIE/13/2363/I01/P, Vol. 1: $416 \mathrm{pp}$.

YaÑEZ-Arancibia, A., DAY J.W. 2004. The Gulf of Mexico: towards an integration of coastal management with large marine ecosystem management. Ocean \& Coastal Management 47: 537-563.

Recibido: 16.08 .2016

Aceptado: 25.01.2018 